\title{
Outcomes of Patients Undergoing Emergency Tracheostomy During COVID 19 Pandemic: Our Experience from a Tertiary Care Centre in North-East India
}

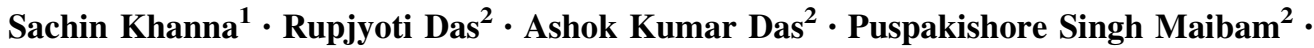 \\ Rohan Dey ${ }^{2}$
}

Received: 30 January 2021 / Accepted: 16 February 2021/Published online: 4 March 2021

(C) Association of Otolaryngologists of India 2021

\begin{abstract}
Airway management in the form of tracheostomy may be done as an emergency or elective procedure depending on when the patient presents and it usually involves a multi-disciplinary team including the anesthesiologist and emergency physician. The purpose of this study is to present our experience with emergency tracheostomies carried out for patients presenting with tumors in the aerodigestive tract during this period of covid 19 pandemic and their outcomes. This is a cross sectional observational study. All the patients who underwent emergency tracheostomy at Dr. B. Borooah Cancer Institute, Guwahati, India, during the period 24th March, 2020 to 23rd September, 2020(6 months) are included in this study. A total of 115 patients underwent 117 emergency tracheostomies (two underwent redo tracheostomies). Median age of patients was 55 years. More than $85 \%$ of patients were male. The most common initial presenting complaint was dysphagia (40\%) followed by dyspnea (25\%). Most common site of tumor was pyriform sinus cancer $(45.2 \%)$. Almost $45 \%$ of patients presented with stage IVA. Complications were seen in 10 patients $(8.7 \%)$. These included stomal stenosis in $4(3.5 \%)$, primary hemorrhage in $3(2.6 \%)$, subcutaneous emphysema, infection and wound break down each in one patient $(0.9 \%$ each). Among the eight residents who performed these procedures, five developed covid 19 symptoms subsequently,
\end{abstract}

Rupjyoti Das

zombiesrubu212@gmail.com

1 Department of Surgical Oncology, Dr B Borooah Cancer Institute, Guwahati, Assam, India

2 Department of Head and Neck Surgery, Dr B Borooah Cancer Institute, House No. 10, Gopal Phukan Path, Ajanta Path, P.O.- Beltola, Kamrup, Guwahati, Assam 781028, India and were tested covid positive. Emergency tracheostomy is a safe procedure for patients; however it carries a higher risk of covid transmission despite using proper precautions.

Keywords Emergency tracheostomy - Covid 19 period . Pandemic

\section{Introduction}

Severe acute respiratory syndrome coronavirus 2 (SARSCoV-2) has become a worldwide pandemic spreading to over 191 countries, and affecting more than sixty-six million people worldwide [1]. Although the majority of individuals experience mild symptoms, approximately 10-17\% develop acute respiratory distress syndrome requiring invasive mechanical ventilation [2, 3]. A nationwide lockdown was imposed by the Government of India from 24th March 2020 to contain the spread of coronavirus infection. This pandemic has caused major disruption of health care services pervading all regions of the world with a calamitous impact on those with limited resources.

Tumors involving the aerodigestive tract can present a great challenge with airway management especially in developing countries like India where large number of patient present at a later stage. Encroachment on the airway may be slow as the tumors grow and acute dyspnea may not be a feature until stridor occurs when airway obstruction is more than $50 \%$ [4].

Airway management in the form of tracheostomy may be done as an emergency or elective procedure [5, 6] depending on when the patient presents and it usually involves a multi-disciplinary team including the anesthesiologist and emergency physician [4]. 
The purpose of this study is to present our experience with emergency tracheostomies carried out for patients presenting with tumors in the aerodigestive tract during this period of covid 19 pandemic and their outcomes.

\section{Materials and Methods}

This is a cross sectional observational study. All the patients who underwent emergency tracheostomy at Dr. B. Borooah Cancer Institute, Guwahati, India, during the period 24th March, 2020 to 23rd September, 2020(6 months) are included in this study. Patient's demographic and clinical profile were noted. The aim of this study is to present the outcomes of these patients during this covid 19 period.

\section{Standard Operating Procedure (SOP)}

Pre procedure covid testing was not mandatory for tracheostomy (as it is a lifesaving emergency procedure), however all patients without pre-operative covid report were kept in isolation ward and followed with covid test (i.e. Rapid Antigen Test (RAT). Personal protection kits (PPE) which included disposable shoe covers, gown, goggles, cap, mask, face shield and gloves were used in every case without pre-tracheostomy covid report. PPE was not used in patients with negative covid test report (RAT) of within seven days of time.

\section{Results}

A total of 115 patients underwent 117 emergency tracheostomies (two underwent redo tracheostomies). Median age of patients was 55 years. More than $85 \%$ of patients were male. Table 1 shows clinico-demographic profile of the patients. The most common initial presenting complaint was dysphagia (40\%) followed by dyspnea (25\%). Maximum number of patients had ECOG (Eastern Cooperative Oncology Group) status of 1(55.6\%).

Most common site of tumor was pyriform sinus cancer $(45.2 \%)$. Almost $45 \%$ of patients presented with stage IVA. Most common histology was squamous cell carcinoma (70.4\%) (Table 2).

Pre-tracheostomy covid testing report was available in 11 patients. Out of that, one was covid positive and rest were negative. On post-tracheostomy covid testing, 9 patients from rest of 104 patients were tested covid positive. They were shifted to dedicated covid ward and managed there till they turned covid negative.

Complications were seen in 10 patients $(8.7 \%)$. These included stomal stenosis in $4(3.5 \%)$, primary hemorrhage in $3(2.6 \%)$, subcutaneous emphysema, infection and wound break down each in one patient $(0.9 \%$ each). The length of hospital stay ranged from 2 to 16 days (mean length of hospital stay was 4.5 days and median was 3 days).

Among the eight residents who performed these procedures, five developed covid 19 symptoms subsequently, and were tested covid positive. All of them recovered well.

\section{Discussion}

Tracheostomy is a surgical procedure that entails making an opening into the trachea and maintaining same with a tube to establish communication with the exterior, [7] the major aim of which is circumventing upper airway obstruction. The most frequent recorded indications for tracheostomy in literature are trauma and head and neck cancers $[8,9]$. In these situations, it is done to relieve upper airway obstructions (either as emergency or elective procedures).

Tracheostomy is an aerosol generating procedure, and health-care workers are at risk of infection during insertion and subsequent care, even when appropriate personal protective equipment (PPE) is used. Aerosol-generating procedures were identified as a leading cause of viral transmission during the SARS outbreak in 2003, with super-spreading events occurring throughout hospitals in Hong Kong, China, and Canada [10]. In our study also, we observed that despite the use of PPE kits and proper guidelines, five out of eight residents who performed these procedures turned covid positive. Although there is no evidence to say that all these covid infections were direct result of tracheostomies.

Table 3 Stage and site wise distribution of patients

\begin{tabular}{llllllll}
\hline Tumor site & $\begin{array}{l}\text { Stage } \\
\text { I }\end{array}$ & $\begin{array}{l}\text { Stage } \\
\text { II }\end{array}$ & $\begin{array}{l}\text { Stage } \\
\text { III }\end{array}$ & $\begin{array}{l}\text { Stage } \\
\text { IVA }\end{array}$ & $\begin{array}{l}\text { Stage } \\
\text { IVB }\end{array}$ & $\begin{array}{l}\text { Stage } \\
\text { IVC }\end{array}$ & Total \\
\hline Hypopharynx & 0 & 2 & 16 & 29 & 5 & 0 & 52 \\
Larynx & 0 & 1 & 7 & 18 & 3 & 0 & 29 \\
Oropharynx & 0 & 1 & 5 & 5 & 2 & 0 & 13 \\
Total & 0 & 4 & 28 & 52 & 10 & 0 & 94 \\
\hline
\end{tabular}

The commonest indication for tracheostomy in our study was hypopharyngeal cancer [45.2\%] (Table 3). This is in contrast to other previous studies that however reviewed tracheostomies done for various other pathologies and different study populations $[8,9]$.

The median age of patients in our experience was 55 years and more than $85 \%$ were males. This is buttressed 
Table 1 Clinico-demographic profile of the patients at initial presentation

\begin{tabular}{ll}
\hline Characteristics & Results \\
\hline Age (Mean) & 54.9 years \\
Age (Median) & 55 years \\
Sex & \\
Male & $98(85.2 \%)$ \\
Female & $17(14.8 \%)$ \\
Presenting complaint & \\
Dysphagia & $46(40 \%)$ \\
Dyspnea & $29(25 \%)$ \\
Hoarseness of voice & $21(18 \%)$ \\
Neck swelling & $21(18 \%)$ \\
Odynophagia & $14(12 \%)$ \\
Stridor & $12(10 \%)$ \\
Foreign body sensation in throat & $11(10 \%)$ \\
Weight loss & $11(10 \%)$ \\
Bleeding from mouth & $5(4 \%)$ \\
ECOG & \\
0 & $16(14 \%)$ \\
1 & $64(55.6 \%)$ \\
2 & $28(24.3 \%)$ \\
3 & $5(4.3 \%)$ \\
4 & $2(1.7 \%)$ \\
\hline
\end{tabular}

Table 2 Tumor characteristics

\begin{tabular}{ll}
\hline Characteristics & Result \\
\hline Tumor site & \\
Hypopharynx & $52(45.2 \%)$ \\
Larynx & $29(25.2 \%)$ \\
Oropharynx & $13(11.3 \%)$ \\
Unknown & $21(18.2 \%)$ \\
Stage & \\
I & $0(0 \%)$ \\
II & $4(3.5 \%)$ \\
III & $28(24.3 \%)$ \\
IVA & $52(45.2 \%)$ \\
IVB & $10(8.7 \%)$ \\
IVC & $0(\%)$ \\
Unknown & $21(18.2 \%)$ \\
Histology & \\
Squamous cell carcinoma & $81(70.4 \%)$ \\
Small cell carcinoma & $1(0.8 \%)$ \\
Lymphoma & $3(2.6 \%)$ \\
Unknown & $30(26 \%)$ \\
\hline
\end{tabular}

by the fact that hypopharyngeal cancer was the commonest indication for the procedure, a disease commonly affecting males in this age group with males about 4 or more times more affected than females [11, 12].

Emergency awake tracheostomy is beneficial in patients that present with impending airway obstruction and is a safe procedure when performed appropriately. [13] The challenge with tracheostomy in such an emergency situation is positioning of the patients (difficulty experienced in supine position with neck extension in their hypoxic state). This makes the procedure difficult and increases risks. [14] In our study we encountered three $(2.6 \%)$ cases of primary hemorrhage, all of them occurred in patients who presented with severe respiratory distress.

We recorded an overall complication rate of $8.7 \%$ in our study (including $2.6 \%$ of primary hemorrhage). The rate of complications following tracheostomy varies from one part of the world to another and ranges of 5-40\% have been reported with these rates increasing 2-5 folds following emergency tracheostomy [15]. Like previous studies documented, we also agree that emergency tracheostomy in patients with aerodigestive tract tumors is a relatively safe but challenging procedure. Most of the complications are due to poor tissue handling in emergency situations, and restlessness of the patient due to air hunger.

The median length of hospital stay in our study was 3 days (mean was 4.5 days and range 2-16 days) indicating the effective post-operative care given to our patients necessitating early hospital discharge. Only covid positive patients had a longer hospital stay as they were kept under observation until they turned covid negative. A shorter hospital stay reduces the risk of nosocomial infections, reduces morbidity and mortality and the financial burden on patients and care-givers [16]. Less invasive procedures like percutaneous dilatational tracheostomy have been reported in recent times with lesser complication rates than open tracheostomy; [17] however, our center lacks facilities for this method of tracheostomy.

Our decannulation rate was $0 \%$. As all of the patients were either under evaluation, receiving definitive treatment, had just finished their treatment course, haven't had their post treatment check endoscopy done yet or needed tracheostomy for palliative care.

The overall hospital mortality rate was $0 \%$ during the study period. However thirty patients were lost to follow up during the period of evaluation, as our part of the country was in lockdown situation.

The limitations of our study included: first, it is a single center study. Secondly it is a observational study and thirdly, it involves a relatively small study population and this may have resulted in a lack of statistically significant correlations limiting interpretation of the relationships between variables. 


\section{Conclusion}

Emergency tracheostomy is a safe procedure for patients; however it carries a higher risk of covid transmission despite using proper precautions. Nonetheless, despite the risks associated, it is a lifesaving procedure and has to be performed when needed. We suggest whenever needed (during this covid period), this procedure should be performed by experienced surgeons with minimal staff wearing personal protective kit.

Funding None.

Availability of Data and Material Accurate data and materials have been collected.

Code Availability Not applicable.

\section{Compliance with Ethical Standards}

Conflict of interest The author(s) declare that they have no competing interests.

Consent to Participate It has been taken.

Consent for Publication It has been taken.

Ethics Approval It has been taken.

\section{References}

1. Johns Hopkins University Coronavirus Resource Center. Coronavirus COVID-19 Global Cases by the Center for Systems Science and Engineering (CSSE) at Johns Hopkins University. Available from: https://coronavirus.jhu.edu/map.html. Accessed 06 Dec 2020

2. Zhou F, Yu T, Du R et al (2020) Clinical course and risk factors for mortality of adult inpatients with COVID-19 in Wuhan, China: a retrospective cohort study. Lancet 395:1054-1062

3. Guan W, Ni Z, Hu Y et al (2020) Clinical characteristics of coronavirus disease 2019 in China. N Engl J Med 382:1708-1720
4. Altman KW, Waltonen JD, Kern RC (2005) Urgent surgical airway intervention: a 3-year county hospital experience. Laryngoscope 115:2101-2104

5. Costa L, Matos R, Julio S, Vales F, Santos M (2016) Urgent tracheostomy: four-year experience in a tertiary hospital. World $\mathrm{J}$ Emerg Med 7:227-230

6. Leiser Y, Barak M, Ghantous Y, Yehudai N, Abu E-N (2017) Indications for elective tracheostomy in reconstructive surgery in patients with oral cancer. J Craniofac Surg 28:e18-e22

7. Adoga AA, Maâ ${ }^{\mathrm{TM}}$ an ND (2010) Indications and outcome of pediatric tracheostomy: results from a Nigerian tertiary hospital. BMC Surg 10:2

8. Bhatti ABH, Iqbal H, Hussain R, Syed AA, Jamshed A (2015) Tracheostomy in cancer patients: experience from a cancer hospital in Pakistan. Indian J Surg 77:906-909

9. Amusa YB, Akinpelu VO, Fadiora SO, Agbakwuru EA (2004) Tracheostomy in surgical practice: experience in a Nigerian tertiary hospital. West Afr J Med 23:32-34

10. Tran K, Cimon K, Severn M, Pessoa-Silva CL, Conly J (2012) Aerosol generating procedures and risk of transmission of acute respiratory infections to healthcare workers: a systematic review. PLoS ONE 7:e35797

11. Perdomo S, Martin Roa G, Brennan P, Forman D, Sierra MS (2016) Head and neck cancer burden and preventive measures in Central and South America. Cancer Epidemiol 44(Supp11):S43S52

12. Nallathambi C, Yumkhaibam SD, Singh LJ, Singh TT, Singh IY, Daniel N (2016) Clinico-epidemiologic patterns of laryngeal cancer: 5-year results from a regional cancer center in Northeastern India. Asian Pac J Cancer Prev 17:2439-2443

13. Fang CH, Friedman R, White PE, Mady LJ, Kalyoussef E (2015) Emergent awake tracheostomy: the five-year experience at an urban tertiary care center. Laryngoscope 125:2476-2479

14. Yuen HW, Loy AH, Johari S (2007) Urgent awake tracheostomy for impending airway obstruction. Otolaryngol Head Neck Surg 136:838-842

15. Goldenberg D, Ari EG, Gotz A, Danino J, Metzer A, Joachims HZ (2000) Tracheostomy complications: a retrospective study of 1130 patients. Otolaryngol Head Neck Surg 123:495-500

16. Kochhar A, Pronovost PJ, Gourin CG (2013) Hospital-acquired conditions in head and neck cancer surgery. Laryngoscope 123:1660-1669

17. Delaney A, Bagshaw SM, Nalos M (2006) Percutaneous dilatational tracheostomy versus surgical tracheostomy in critically ill patients: a systematic review and meta-analysis. Crit Care 10:R55

Publisher's Note Springer Nature remains neutral with regard to jurisdictional claims in published maps and institutional affiliations. 